\title{
BMJ Open Protocol for a realist review of complex interventions to prevent adolescents from engaging in multiple risk behaviours
}

\author{
Christina Cooper, ${ }^{1}$ Monique Lhussier, ${ }^{1}$ Janet Shucksmith, ${ }^{2}$ Susan Mary Carr ${ }^{1}$
}

To cite: Cooper C, Lhussier M, Shucksmith J, et al. Protocol for a realist review of complex interventions to prevent adolescents from engaging in multiple risk behaviours. BMJ Open 2017;7:e015477. doi:10.1136/ bmjopen-2016-015477

- Prepublication history and additional material for this paper are available online. To view these files please visit the journal online (http://dx.doi. org/10.1136/bmjopen-2016015477).

Received 3 January 2017 Revised 17 July 2017 Accepted 25 July 2017

\section{CrossMark}

${ }^{1}$ Faculty of Health and Life Sciences, Northumbria University, Newcastle upon Tyne, UK

${ }^{2}$ Institute of Health and Social Care, University of Teesside, Middlesbrough, UK

Correspondence to Christina Cooper; christina.cooper@northumbria. ac.uk

\section{ABSTRACT}

Objectives Adolescent risk behaviours are a key health concern. The purpose of this research is to gaina deeper understanding of how, why, for whom, and inwhat circumstances complex adolescent risk behaviourprevention programmes are most successful. Methods and analysis To understand how adolescent risk behaviour prevention programmes work in a reallife context, a realist synthesis will be undertaken, operationalised in four phases.

1. Phase one Developing a framework to map the theoretical and conceptual landscape of adolescent risk behaviour prevention. Guided by stakeholder consultation

2. Phase two Formulating initial programme theories through exploration of the literature, along with primary data from professional stakeholder interviews.

3. Phase three Refining programme theories through more purposeful, in depth screening of the literature, along with primary qualitative data, from young people and professionals. Data will be collected through semi structured focus groups, to explore specific elements of the emerging programme theories.

4. Phase four Testing programme theories through interviews with youth workers, following consultation with young people, using vignettes to explore the relationship between specific programme theories. This relatively novel method of primary and secondary data integration within a realist synthesis will provide deeper insight in to young peoples lived experience of risk behaviour prevention programmes, while maintaining transparency in the process of programme theory development.

Data analysis A realist logic of analysis will be used to align data from each phase with context mechanism outcome configurations or specific elements thereof. Substantive theory will then be sought to understand and explain the findings.

Ethics and dissemination This study has been approved by the Ethics committee at Northumbria University, UK. Findings will be disseminated through knowledge exchange with stakeholders, publications in peer-reviewed journals, conference presentations, and formal and informal reports.

\section{BACKGROUND}

Adolescence is typically defined by age, with most studies focusing on the range between
Strengths and limitations of this study

- Uses realist methods to explore contextual factors and underpinning causal mechanisms of complex adolescent risk behaviour prevention.

- Guided by adolescent and professional stakeholders.

- Decision-making is transparent.

- Informs future policy and practice.

- Findings must be considered within the context of this research.

- Formulation of the theoretical framework relies heavily on published, peer-reviewed journal articles, and therefore may be subject to publication bias.

12 (average onset of puberty) and 19 years old. However, the age of participants in studies may range from 10 to 24 years of age. ${ }^{1}$ Arnett $^{2}$ states that the lowering average age of puberty onset; longer time in education; and later achievement of life events such as career, home ownership, marriage and childbirth, result in an extended period of adolescence. For this study, a broad age definition of adolescence will be used to consider the possible impact of age on the adoption of risky behaviours and perceptions of prevention programmes.

Recent research shows that it is important to consider adolescence as a distinct life phase, as well as within the life trajectory. ${ }^{3}$ Adolescence is a key developmental stage with major neurobiological, cognitive, social and behavioural changes occurring. Neurobiological changes such as decline in grey matter (cell volume), number of synapses and density of neurotransmitter receptors, increased white matter (nerve cell axons) leading to faster synapse connectivity and changes in balance between the frontal and limbic systems, ${ }^{4}$ occur in tandem with behavioural and sociological changes, such as identity development and exploration, a drive to become autonomous, while 
maintaining good familial relationships and recognition of the importance of social capital, through the formation and maintenance of relationships with peers, school and the wider community. ${ }^{5}$ This research will explore psychosocial risk behaviour prevention programmes; therefore biological and neurological factors will not be covered in depth. However, it remains important to consider how these changes may impact on adolescent behaviour and interpretation of risk behaviour prevention programme components.

Intervention programmes targeting adolescent risk behaviours have become highly visible, both in policy and practice. ${ }^{6}$ However, only in the last few years have adolescents become the focus of social policy, with many intervention programmes prior to this focusing on younger children or adults, with less emphasis on the social determinants of health during adolescence. ${ }^{1}$ Social determinants of health may be understood on two broad levels as per Sawyer et al. ${ }^{1}$ Structural determinants are factors such as national wealth and inequality, access to education and healthcare, employment opportunities and sex/ gender inequalities. Proximal determinants are factors such as social connectedness within the community, education, family or peer groups. Similar to the concept of proximal determinants of health, is that of individual risk and protective factors. These factors occur at an individual level, such as personality, intelligence and sexual orientation, operating within, and interacting with social determinants of health, and impacting on the likelihood to engage in health risk behaviours. Social determinants of health have an impact throughout the life span, but are thought to become especially salient during adolescence, as children grow into young adults and strive for autonomy. ${ }^{1}$

Although adolescence is typically a healthy life stage, health risk behaviours, such as alcohol consumption, smoking, substance misuse and risky sexual behaviours ${ }^{7}$ have been highlighted as a cause for concern. Evidence suggests that many risk behaviours begun in adolescence impact significantly on longer-term health and wellbeing. ${ }^{8}$ Poor longer-term outcomes include long-term substance abuse, poor physical, mental and sexual health, and lower educational and occupational achievement, making this critical transitional period a key focus for public health programmes.

Until recently, public health behavioural change research has had a tendency to focus on single risk behaviours, for example, alcohol consumption, ${ }^{9}$ substance misuse, ${ }^{1011}$ risky sexual behaviour ${ }^{12} 13$ and smoking. ${ }^{14} 15$ However, adolescent risk behaviours rarely occur in isolation, and there is a growing body of evidence to suggest it may be more relevant to look at a broader range of risk behaviours concurrently, in order to understand common underpinning causal mechanisms and interactions. ${ }^{16}{ }^{17}$ Gateway theories, which propose that participation in one risk behaviour can increase the likelihood of engagement with another, provide further support for this approach. ${ }^{16}$ The key issue in understanding these multiple risk behaviour prevention programmes is that of complexity. Unlike the single focus, brief interventions above, complex programmes target multiple risk behaviours, are often multilevel, and can involve whole institutions or systems. ${ }^{18}$ Furthermore, outcomes are less likely to be one simple measure, but a complex array of broad and sustainable changes across a range of behaviours.

While prevention and intervention programmes have been somewhat effective in reducing risk behaviours, evidence of the effectiveness of complex risk behaviour prevention programmes is highly variable. ${ }^{17}$ Furthermore, evidence from adolescent risk behaviour prevention programmes tends to be programme or theory specific, with little consideration given to young people's personal identity, social networks and feelings of belonging within the community, ${ }^{19}$ and we are therefore potentially missing insights regarding commonly occurring underpinning causal or contextual factors.

This research employs a realist approach, ${ }^{20}$ to investigate how, when and in what circumstances risk behaviour prevention programmes succeed (or fail) in reducing multiple risk behaviours in adolescents. A realist synthesis allows us to source, and synthesise evidence from a broad range of data sources across the adolescent health field, to explain how, why, when and in what circumstances complex multiple risk behaviour prevention programmes succeed or fail. Providing novel insights into adolescent risk behaviour programme development and delivery and allowing us to explore the role and impact of personal, interpersonal and societal factors. ${ }^{19}$

\section{METHODOLOGY}

Realism is a theory-driven methodological paradigm, rooted in philosophy, which sits between positivism (the world is real and can be observed directly) and constructivism (given that all we know has been processed through the human mind, we can never be sure exactly what reality is). Realism posits that social reality cannot be measured directly, but can be understood through careful and systematic investigation of underlying causal mechanisms, the contexts in which events occur, and the outcomes produced. Complex health interventions do not act as an independent agent for change, but rather operate within open systems, interacting with personal, interpersonal and environmental factors outside of the programme. ${ }^{1821}$ While these factors are usually controlled for within the experimental paradigm, realism seeks to explore and understand how these interactions impact on programme success. This is achieved through the development, testing and refining of a realist programme theory, consisting of context, mechanism, outcome configurations (CMOCs) ${ }^{22}$ By understanding the CMOCs within a programme, we can understand how, why, for whom, in what contexts and how it produces its desired and undesired outcomes in the short, medium and long term. 
From a realist perspective, programme theory describes the development and implementation of a prevention programme from the conception, in the minds of policy-makers, academics and programme developers, through practitioners and managers involved in programme delivery, into the minds of the service user or participant. ${ }^{18}$ At each stage, the programme may be interpreted and/or implemented differently, with possible impact on outcomes. Realist methodologies aid the development of a broader picture of how such combinations of context and underlying causal mechanisms can improve or impair programme fidelity and efficacy.

\section{REVIEW OBJECTIVES}

The aims of the review are the following:

1. To use a theory-driven approach to identify factors which influence the success or failure of complex adolescent risk behaviour prevention programmes in reducing adolescent risk behaviours.

2. To produce a set of refined programme theories of causal mechanisms and contextual factors which operate within strategies to facilitate change across short-term, medium-term and long-term outcomes.

3. To produce guidelines based on the evidence synthesis for consideration in future development and use of adolescent risk behaviour prevention programmes in research, policy and practice.

\section{RESEARCH QUESTIONS}

What are the key contextual factors which influence the success or failure of complex prevention programmes for adolescent's risk behaviours?

What are the key underpinning mechanisms which, in the right contexts, lead to the success or failure of complex adolescent risk behaviour prevention programmes?

Are contextual factors and causal mechanisms consistent across a range of risk behaviours?

How do broader contextual factors impact on programme specific programme theories?

How might this influence future research, policy and practice?

\section{METHODS}

The inclusion of service users and stakeholders in healthcare decision-making and research, is becoming increasingly popular ${ }^{23}$ and has long been advocated in a wide range of policies. ${ }^{24}$ While close stakeholder engagement, from the outset, is recommended in realist synthesis, ${ }^{182526}$ the fact it is considered here as primary data, and the way in which data will be analysed, coded and integrated, from both primary and secondary sources, within a realist synthesis, is novel. The planned research will use this combination of primary and secondary data to explore developing programme theories. As this research aims to investigate what works, for whom and in what circumstances across a broad range of programmes, delivered in a wide range of settings, we propose that the inclusion of primary data in the synthesis will give greater insight into possible causal mechanisms which might not be apparent within the literature. It will provide additional transparency in the process of developing, evidencing, refining, adjudicating between and refuting emerging programme theories. Furthermore, we propose that the inclusion of primary data will give greater strength to stakeholder voices within the research, particularly those of adolescents themselves. ${ }^{19}$ Given the multifaceted approach planned, the research is described here in four distinct phases in order to maintain clarity. However, the synthesis process is much more iterative, cycling between empirical literature searching and data collection, and constant refinement of, adjudication between, and evidencing of emerging programme theories represented here in figure 1 in online supplementary material (Developed from descriptions by Nick Emmel in his book Sampling and choosing cases in qualitative research: A realist approach). ${ }^{27}$ This constant triangulation will continue throughout the research period.

\section{Phase 1: Developing the theoretical framework}

At the time of submission, phase 1 of the research has been conducted, to guide and develop the research protocol.

Early screening of the literature investigates the theoretical underpinnings on which programmes are based in order to map out the conceptual and theoretical landscape of adolescent risk behaviour programmes, how they are supposed to work, and at what points intended protocol is changed or adapted to suit circumstance. ${ }^{18}$ Literature searching was carried out using search terms defined by the research questions, for example, adolescent risk behaviour prevention, complex risk behaviour prevention programmes (see online supplementary material for search terms).

Development of the initial research questions and development of the theoretical framework were carried out in consultation with a young person's advisory group. The panel is a pre-existing group of young people, both men and women, aged 13-19 years. The purpose of the panel, accessed through the Fuse Centre for Translational Research (www.fuse.ac.uk), is to provide advice and guidance to researchers carrying out research which involves or impacts on young people. The group were also consulted over the development of research materials to be used by their peers within this project. This approach benefits from experiential knowledge, recognising young people as experts in adolescent life, ensuring materials are accessible, and produce relevant and meaningful findings. ${ }^{28}$

This highlighted some broad factors which may impact on adolescent risk behaviour prevention programme outcomes. These broad themes, are at this stage indicative only, providing a framework from which candidate programme theories can be developed: 


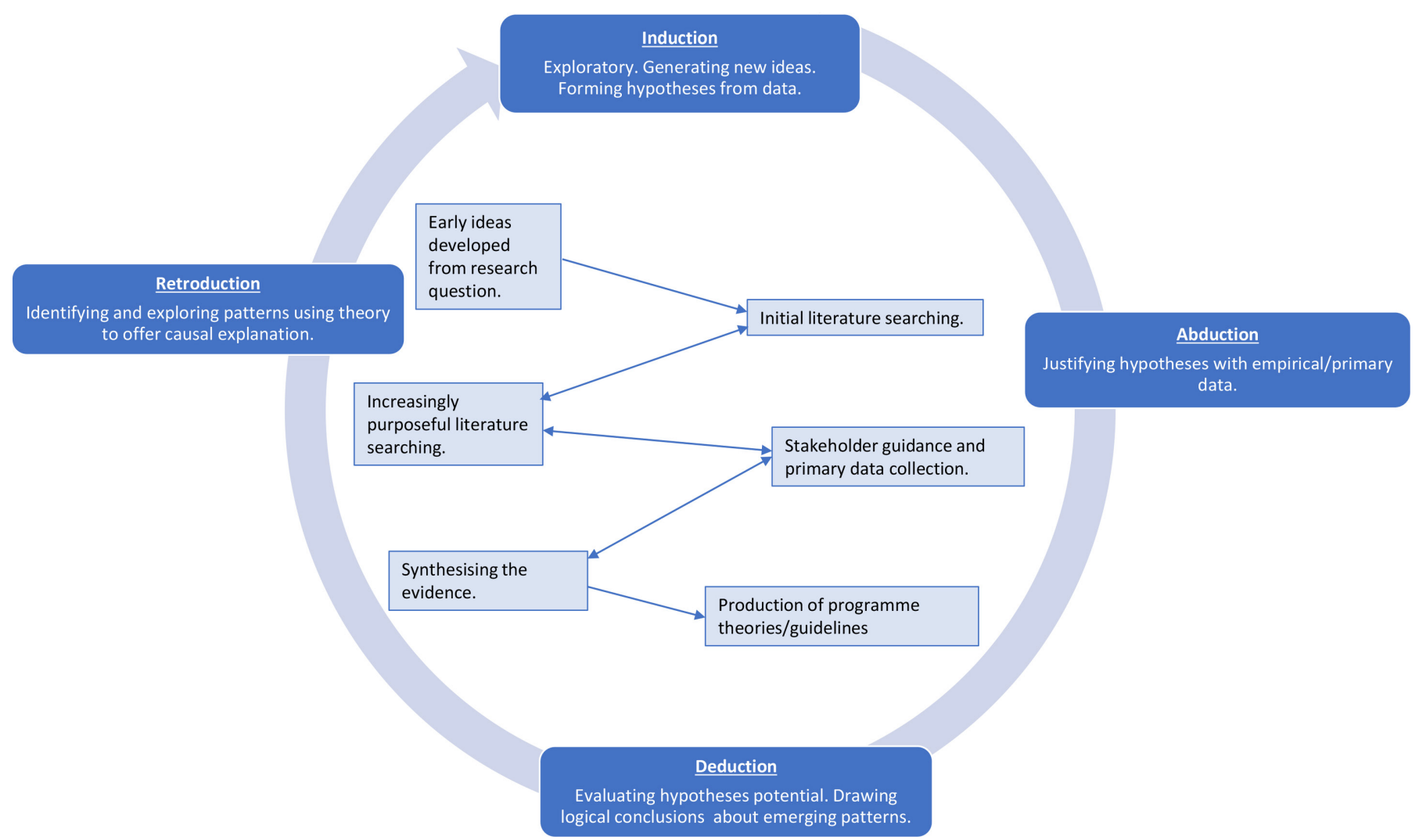

Figure 1 Zigzagging-Realist synthesis data collection processes.

- Training and resources which are required and/or provided by the programme;

- Mode of delivery;

- Location for delivery (school, community, family);

- Attitudes and beliefs of those supporting the programme;

- Attitudes and beliefs of those delivering the programme;

- Content/behavioural change techniques employed by the programme;

- Social connectedness;

- Familial influence;

- Individual factors (resilience, self-efficacy, autonomy).

\section{Phase 2: Formulating initial programme theories}

The purpose of the review at this stage is to investigate demi-regularities in outcome patterns, exploring how or why programmes may have succeeded in one instance but failed in another, or why actual outcomes do not meet with expected outcomes. It is this questioning of how, why and in what circumstances programmes produce both intended and unintended outcomes that allows us to consider the underpinning causal mechanisms, and contextual factors which may impact on them.

\section{Literature search strategy}

Consistent with the realist approach, there are several search phases throughout the evidence synthesis, starting with a broad search to allow the development of a theoretical framework (Phase 1), becoming more specific as evidence is sought to formulate, support, adjudicate between, or discard potential or emergent programme theories. Literature searching here focusses primarily on evidence from a range of primary empirical studies investigating complex adolescent risk behaviour prevention programmes. Search terms are defined by the theoretical framework, as set out in phase one (see online supplementary material).

The databases to be searched include, but are not be restricted to: Applied Social Science Index Abstracts , Child Development and Adolescent Studies, CINAHL, Cochrane, MEDLINE, PsychArticles and Web of Science. Other sources may be used to identify further evidence from grey literature and web pages. A comprehensive list of search terms and databases used will be provided in subsequent publications on completion of the proposed research.

\section{Inclusion and exclusion criteria}

Broad inclusion/exclusion criteria, as set out in the table 1, were used to guide the scope of literature searching in the early phases of the research.

Formulation of the initial programme theories is supported by professional stakeholder interviews. Consisting of a minimum of six professional stakeholders from the adolescent health field, including researchers, teachers, community youth workers, PHSE leaders, peer coordinators and school nurses. Recruitment of the professional stakeholders will use an approach 


\section{Inclusion criteria}

1. Programmes targeting adolescents aged between 10 and 24 years.

2. Programmes targeting two or more risk behaviours (alcohol misuse, risky sexual behaviours, smoking, substance misuse).

3. Complex risk behaviour prevention programmes (using a range of behaviour change techniques, on several levels, across contexts), designed to be delivered to the general population.

4. Complex multiple risk behaviour prevention programmes based on a psychosocial model of behaviour change.

\section{Exclusion criteria}

1. Programmes exploring interventions in childhood or adulthood.

2. Programmes designed to target one specific risk behaviour only.

3. Simple/brief/targeted interventions, designed for use with specific, at risk, populations.

4. Programme with a biological/neurological focus. which is both theoretical and purposive, to allow recruitment of those with expert knowledge in areas relevant to the emerging programme theories, to aid in identifying, developing and prioritising emerging programme theories. ${ }^{27}$ Recruitment will continue throughout the research, and participants may be consulted on more than one occasion to enable better understanding of emerging programme theories. Consultations will consist of interviews, conducted face-to-face or via video calling. All participants are provided with a detailed information sheet, and informed consent obtained prior to interviewing. Interviews are audio-recorded and transcribed verbatim. Data will aid in uncovering how emerging mechanistic and contextual factors may contribute to demi-regularities seen in outcome patterns emerging from the empirical literature. Furthermore, data may also be used to identify possible new contexts and/or mechanisms for exploration in the literature.

\section{Phase 3: Refining programme theories}

This phase seeks to address the following issues ${ }^{18}$ (examples given below are potential programme theories from early phases of the research):

1. Reviewing programme theory integrity

Adolescent risk behaviour prevention programmes tend to be complex, with a number of stages or levels, within the programme, at which change or deviation from the intended programme may impact on outcomes. The purpose of this strategy is to explore weak points which commonly occur in the history of implementation of adolescent risk behaviour prevention programmes.

Within this research this will, for example, involve consideration of how training and resources are made available to the deliverer, who delivers the programme, mode and location of delivery impact on programme outcomes. Evidence for this will largely be sought from the empirical and theoretical literature as well as through consultation with programme developers and deliverers.

For example: Complex adolescent risk behaviour prevention programmes delivered by a facilitator (c) are more successful when good quality programme delivery training is provided, including leadership/teaching skills ( $\mathrm{M}$ resource), ensuring facilitators have a clear understanding of the programme strategies and behavioural change techniques contained within the programme, and how to deliver them (M reasoning), ensuring programme components are delivered well (o).

Intervention programmes which adhere to the intended delivery strategy (c) provide clear information, support and opportunities for skills development ( $\mathrm{M}$ resource) making it easy for adolescents to access, understand and use programme strategies (M reasoning) increasing the likelihood of a change in beliefs or behaviour (o).

2. Reviewing to adjudicate between rival theories

The purpose of this strategy is to gather evidence to discover which, of a number of competing programme theories, is driving differences in programme outcomes.

Frequently, within the literature, it is not possible to elicit which, of a number of possible underlying causal factors is driving changes in outcome. This is a commonly occurring problem in the literature making consultation with a range of relevant stakeholders invaluable in clarifying what works, for whom and in what circumstances.

For example: Adolescent risk behaviour prevention programmes designed to change beliefs around social norms (c) are more successful in changing attitudes and beliefs $(\mathrm{o})$ when delivered by a peer educator $(\mathrm{M}$ resource) as young people are more likely to identify with the beliefs of their peers ( $M$ reasoning). OR Adolescent risk behaviour prevention programmes designed to change beliefs around social norms (c) are more successful in changing attitudes and beliefs (o) when delivered by a peer educator (resource) as adolescents are more open to communication with peers and therefore engage more fully with the programme components (reasoning).

3. Reviewing the same theory in comparative settings The purpose of this strategy is to further explore for whom and in what circumstances a programme is successful. This method tends to focus on specific components of the prevention programme, looking for techniques or components of the programme that are more or less successful within each context. 
This type of component by component comparison will also be used to explore how, for whom and in what circumstances specific components of a range of prevention programmes with differing theoretical underpinning impact on outcomes. It is believed that data from young people themselves will be key in developing greater understanding here.

For example: Risk behaviour prevention programmes delivered in schools, which take a harm reduction approach $(\mathrm{C})$, containing advice and information (signposting) on what to do if you do have a problem or are participating in risky health behaviours ( $\mathrm{M}$ resource), are more likely to succeed in changing behaviour (o) than those which take a 'should not' 'do not' approach as young people felt their needs were actually being considered and were less likely to switch off to a 'telling off' (M reasoning).

\section{Literature search strategy}

In accordance with the realist approach, searching in this phase will locate evidence from a broad range of sources including empirical efficacy studies, editorials, evaluations, systematic reviews and follow-up papers. Literature searches will be guided by the review objectives and initial programme theories, along with any new causal mechanisms and contextual factors emerging from stakeholder interviews in phase 2. Here, as evidence sought relates to causal mechanisms and/or contextual factors, rather than programmes, evidence may be sourced from literature which crosses programme, sector and/disciplinary boundaries. ${ }^{29}$ Therefore, at this stage literature searching will not be guided by the inclusion/exclusion criteria of the previous stage. Increasingly purposive searches will be carried out in relation to emerging theories or areas of explanatory potential. A number of methods recommended for realist evidence searching will be used throughout. ${ }^{30} 31$ These include using new, targeted search terms not defined in the initial search, and reference and citation searches of key papers to identify parent, sibling and follow-up papers which relate to emerging programme theories.

\section{Quality appraisal}

Papers will be appraised using an adapted tool based on previous appraisal work within a realist project. The tool allows papers to be appraised for rigour and relevance, and ranked as conceptually rich (high), moderate or low. Evidence will not be excluded based on this appraisal, but it will allow a focus on the more conceptually rich papers, without excluding weaker papers which may still contribute to the final evidence synthesis. ${ }^{32}$

It is widely acknowledged in realist research ${ }^{18}{ }^{29}$ that evidence of causal mechanisms, particularly end user reasoning in engaging with programme resources, is difficult to unearth through investigation of the published literature. For this reason, the inclusion of primary data from stakeholders, will bring added value to the research.

Primary data will be collected from seven focus groups in total.
Five focus groups with young people: a mixed gender group, a mixed gender group from low socioeconomic backgrounds (as determined by postcode), a girls only group, a boys only group and a mixed group of those who have accessed services for health information.

Groups will be selected using purposive sampling to ensure a broad range of experience, and reduce the impact of health inequalities, based on gender or socioeconomic status. Young people will be recruited, via email to gatekeepers through local schools, services and youth groups across the North East of England. Interviews will be conducted within the participating groups usual surroundings (youth club, school, council offices). All participants will complete a participant information sheet and an informed consent form prior to data collection.

Focus groups will employ a semistructured interview technique, with questions guided by emerging programme theories from the early phases of the research. In addition to this young people will be provided with large sheets of paper, sticky notelets and pens, to make the research as accessible as possible, allowing young people to choose the way in which they find it most comfortable to express their opinions. ${ }^{33}$ Focus groups will be transcribed verbatim, and data will be analysed, alongside written and pictorial evidence produced.

Two focus groups with school nurses: school nurses will be recruited using opportunity sampling, based on availability to participate at the time of carrying out the research. All participants will provide informed consent prior to interviewing. Interviews will be conducted within a local health centre.

A semistructured interview technique will be employed. Questions were guided by emerging programme theories from the early phases of the research. Focus groups will be transcribed verbatim.

\section{Data extraction}

Extraction of the data will be recorded using a specially developed data extraction table to record the data, and aid in the process of sorting, sifting and annotating data source materials. Thematic analysis and coding techniques will be employed to track which data sources evidence which elements of which programme theories. Data extraction from both primary and secondary data sources will be undertaken simultaneously to allow constant triangulation across all data sets.

\section{Phase 4: Testing programme theories}

Young people are often thought of as a vulnerable, hard to reach population. In order to gather the opinion of as many young people as possible, with minimal risk, a series of vignettes will be designed to investigate relationships between key underpinning causal mechanisms and contextual factors from refined programme theories. Vignettes will be used to provide a common context around which discussion may be shaped, reducing the need to rely on a personal frame of reference, allowing young to talk openly and without judgement. ${ }^{19}$ 
Purposive sampling will be used to recruit two local community youth groups providing services for young people aged 12-24 years. Participation in the research will involve two meetings between the researcher and youth group leaders, and a discussion between the leader and the young people about risk behaviour prevention, based on a set of vignettes. Data will be collected during the second meeting, which will involve youth group leaders feeding back the results of the discussion to the researcher, in a recorded interview.

Using youth leaders, who have an existing, trusting relationship with the young people, as facilitators in this way can reduce the impact of perceived power imbalances between researcher and participant. Researcher bias is also reduced ${ }^{32}$ increasing the likelihood that the data gathered is as representative of young people's opinions as possible.

Interviews will be recorded and transcribed verbatim. Data from the interviews will then be used to further evidence the refined programme theories from earlier stages, and to aid in understanding relationships between theories in the final analysis.

\section{DATA ANALYSIS}

Data analysis following a realist logic can be broken down in to two key tasks. The first involves aligning the evidence with specific elements of the refined programme theories to show that particular mechanisms produce certain outcomes, within a specific context. The aim here is to explain whether and to what degree, mechanisms are activated within a particular context to produce an outcome or set of outcomes, using cross-programme, inter-context comparisons to draw out patterns of demi-regularity from the literature, and supported here by the data collected.

The final task in realist synthesis involves drawing on substantive theory in order to make sense of the pattern of findings. ${ }^{18} 29$

The data synthesis will be carried out using NVIVO to collate, annotate and align evidence from primary and secondary data, and to map out relationships within and between refined programme theories, supported by substantive theory.

\section{DISSEMINATION}

The realist synthesis approach allows use of the existing literature, along with new evidence from primary data to provide a novel insight into how, when and in what circumstances complex adolescent multiple risk behaviour prevention programmes succeed or fail. Involvement of stakeholders in the research process gives the opportunity for knowledge transfer throughout. Furthermore, the aim of the research is to inform future policy, research and practice, in the development and delivery of complex multiple risk behaviour programmes. Findings from the research will be disseminated on a broader basis through publication in peer-reviewed journals, conferences, and formal and informal presentations and reports.

Contributors All authors contributed to conceptualisation of the study, with leadership from CC and ML. CC wrote the first draft. ML, JS and SMC critically reviewed it and provided comments to improve the manuscript. All authors have read and approved the final manuscript.

Funding The project is funded by the Medical Research Council (MRC) through Fuse Centre for Translational Research in Public Health.

Competing interests None declared.

Ethics approval Northumbria University ethics committee.

Provenance and peer review Not commissioned; externally peer reviewed.

Open Access This is an Open Access article distributed in accordance with the Creative Commons Attribution Non Commercial (CC BY-NC 4.0) license, which permits others to distribute, remix, adapt, build upon this work non-commercially, and license their derivative works on different terms, provided the original work is properly cited and the use is non-commercial. See: http://creativecommons.org/ licenses/by-nc/4.0/

C Article author(s) (or their employer(s) unless otherwise stated in the text of the article) 2017. All rights reserved. No commercial use is permitted unless otherwise expressly granted.

\section{REFERENCES}

1. Sawyer SM, Afifi RA, Bearinger $\mathrm{LH}$, et al. Adolescence: a foundation for future health. Lancet 2012;379:1630-40.

2. Arnett JJ. Emerging adulthood. A theory of development from the late teens through the twenties. Am Psychol 2000;55:469-80.

3. Patton GC, Coffey C, Cappa C, et al. Health of the world's adolescents: a synthesis of internationally comparable data. Lancet 2012;379:1665-75.

4. Blakemore SJ. Imaging brain development: the adolescent brain. Neuroimage 2012;61:397-406.

5. Syed M, Seiffge-Krenke I. Personality development from adolescence to emerging adulthood: linking trajectories of ego development to the family context and identity formation. $J$ Pers Soc Psychol 2013;104:371-84.

6. Fuller E, Smoking, drinking and drug use among young people in England in 2011. London: Health and Social Care Information Centre, 2012.

7. Hagell A, Coleman J, Brooks F. Key data on adolescence. London: Association for Young People's Health, 2013.

8. Kipping RR, Smith M, Heron J, et al. Multiple risk behaviour in adolescence and socio-economic status: findings from a UK birth cohort. Eur J Public Health 2015;25:44-9.

9. Schelleman-Offermans K, Knibbe RA, Kuntsche E. Preventing adolescent alcohol use: effects of a two-year quasi-experimental community intervention intensifying formal and informal control. J Adolesc Health 2014;54:326-32.

10. Botvin GJ, Griffin KW, Diaz T, et al. Drug abuse prevention among minority adolescents: posttest and one-year follow-up of a schoolbased preventive intervention. Prev Sci 2001;2:1-13.

11. Schwinn TM, Schinke SP, Di Noia J. Preventing drug abuse among adolescent girls: outcome data from an internet-based intervention. Prev Sci 2010;11:24-32.

12. Caruthers AS, Van Ryzin MJ, Dishion TJ. Preventing high-risk sexual behavior in early adulthood with family interventions in adolescence: outcomes and developmental processes. Prev Sci 2014;15 (Suppl 1) $59-69$.

13. Jemmott JB, Jemmott LS, Fong GT. Efficacy of a theory-based abstinence-only intervention over 24 months: a randomized controlled trial with young adolescents. Arch Pediatr Adolesc Med 2010;164:152-9.

14. Campbell R, Starkey F, Holliday J, et al. An informal school-based peer-led intervention for smoking prevention in adolescence (ASSIST): a cluster randomised trial. Lancet 2008;371:1595-602.

15. Simons-Morton BG, Farhat T. Recent findings on peer group influences on adolescent smoking. J Prim Prev 2010;31:191-208.

16. Hale DR, Fitzgerald-Yau N, Viner RM. A systematic review of effective interventions for reducing multiple health risk behaviors in adolescence. Am J Public Health 2014;104::e19-e41.

17. Jackson CA, Henderson M, Frank JW, et al. An overview of prevention of multiple risk behaviour in adolescence and young adulthood. J Public Health 2012;34(suppl 1):i31-i40. 
18. Pawson R, Greenhalgh T, Harvey G, et al; Realist synthesis: an introduction. ESRC research methods programme. Manchester: University of Manchester, 2004.

19. Morrow V. Ethical dilemmas in research with children and young people about their social environments. Child Geogr 2008;6:49-61.

20. Pawson R, Tilley N. An introduction to scientific realist evaluation. London: Sage, 1997

21. Connelly JB. Evaluating complex public health interventions: theory, methods and scope of realist enquiry. J Eval Clin Pract 2007;13:935-41.

22. Pawson R. Evidence-based policy: a realist perspective. London: Sage, 2006.

23. Thompson AG. The meaning of patient involvement and participation in health care consultations: a taxonomy. Soc Sci Med 2007;64:1297-310.

24. Groene O, Garcia-Barbero M, eds. Health promotion in hospitals: evidence and quality management. Copenhagen: WHO Regional Office for Europe, 2005.

25. Rycroft-Malone J, Burton C, Hall B, et al. Improving skills and care standards in the support workforce for older people: a realist review. BMJ Open 2014;4:e005356.
26. Lhussier M, Carr SM, Forster N. A realist synthesis of the evidence on outreach programmes for health improvement of Traveller Communities. J Public Health 2016;38:e125-e132.

27. Emmel N. Sampling and choosing cases in realist research: a realist approach. London: Sage, 2013.

28. Moore T, Noble-Carr D, McArthur M. Changing things for the better: the use of children and young people's reference groups in social research. Int J Soc Res Methodol 2016;19:241-56.

29. Wong G, Greenhalgh T, Westhorp G, et al. RAMESES publication standards: realist syntheses. BMC Med 2013;11:21.

30. Papaioannou D, Sutton A, Carroll C, et al. Literature searching for social science systematic reviews: consideration of a range of search techniques. Health Info Libr J 2010;27:114-22.

31. Finfgeld-Connett $D$, Johnson ED. Literature search strategies for conducting knowledge-building and theory-generating qualitative systematic reviews. J Adv Nurs 2013;69:194-204.

32. Pawson R. Digging for nuggets: how 'bad' research can yield 'good' evidence. Int J Soc Res Methodol 2006;9:127-42.

33. Morrow V. Using qualitative methods to elicit young people's perspectives on their environments: some ideas for community health initiatives. Health Educ Res 2001;16:255-68. 